\title{
Tunable Mega-Ampere Electron Current Propagation in Solids by Dynamic Control of Lattice Melt
}

\author{
D. A. MacLellan, ${ }^{1}$ D. C. Carroll, ${ }^{1}$ R. J. Gray, ${ }^{1}$ N. Booth, ${ }^{2}$ M. Burza, ${ }^{3}$ M. P. Desjarlais, ${ }^{4}$ F. Du, ${ }^{5}$ D. Neely, ${ }^{2}$ H. W. Powell, ${ }^{1}$ \\ A. P. L. Robinson, ${ }^{2}$ G. G. Scott, ${ }^{1,2}$ X. H. Yuan, ${ }^{6}$ C.-G. Wahlström, ${ }^{3}$ and P. McKenna, ${ }^{1, *}$ \\ ${ }^{1}$ SUPA, Department of Physics, University of Strathclyde, Glasgow G4 ONG, United Kingdom \\ ${ }^{2}$ Central Laser Facility, STFC Rutherford Appleton Laboratory, Oxfordshire OX11 OQX, United Kingdom \\ ${ }^{3}$ Department of Physics, Lund University, P.O. Box 118, S-22100 Lund, Sweden \\ ${ }^{4}$ Sandia National Laboratories, P.O. Box 5800, Albuquerque, New Mexico 87185, USA \\ ${ }^{5}$ Beijing National Laboratory of Condensed Matter Physics, Institute of Physics, Chinese Academy of Sciences, Beijing 100190, China \\ ${ }^{6}$ Key Laboratory for Laser Plasmas (Ministry of Education) and Department of Physics and Astronomy, \\ Shanghai Jiao Tong University, Shanghai 200240, China \\ (Received 23 June 2014; published 31 October 2014)
}

\begin{abstract}
The influence of lattice-melt-induced resistivity gradients on the transport of mega-ampere currents of fast electrons in solids is investigated numerically and experimentally using laser-accelerated protons to induce isochoric heating. Tailoring the heating profile enables the resistive magnetic fields which strongly influence the current propagation to be manipulated. This tunable laser-driven process enables important fast electron beam properties, including the beam divergence, profile, and symmetry to be actively tailored, and without recourse to complex target manufacture.
\end{abstract}

DOI: 10.1103/PhysRevLett.113.185001

PACS numbers: $52.38 . \mathrm{Kd}$

The propagation of mega-ampere currents of fast (multi$\mathrm{MeV}$ ) electrons in solids driven by relativistically intense laser pulses (peak intensity, $I_{L}>10^{18} \mathrm{~W} \mathrm{~cm}^{-2}$ ) underpins numerous applications of high power lasers, including the fast ignition approach to inertial confinement fusion [1] and the generation of intense sources of $\mathrm{x}$ rays [2], ions [3], and pair production [4]. Many of these applications require control of the fast electron beam divergence and thus the role of self-generated resistive magnetic fields [5] in pinching or collimating the electron beam has been explored. The magnetic field growth rate is given by $\partial \mathbf{B} / \partial t=\eta \nabla \times \mathbf{j}_{f}+\nabla \eta \times \mathbf{j}_{f}$, where $\eta$ is the background electrical resistivity and $\mathbf{j}_{f}$ is the fast electron current density. The first term on the right forces electrons into regions of higher current density, while the second seeds magnetic field growth in regions of resistivity gradients, driving electrons towards regions of higher resistivity. Several schemes involving complex, premanufactured targets with regions containing resistivity boundaries at the interface of layered materials have been explored with the aim of manipulating fast electron transport [6-11]. The potential to use ionization variations in homogeneous metal targets to create desired resistivity profiles, and, hence, resistive magnetic field patterns, has also been explored [12].

Published by the American Physical Society under the terms of the Creative Commons Attribution 3.0 License. Further distribution of this work must maintain attribution to the author(s) and the published article's title, journal citation, and DOI.
Recently, it was shown that lattice structure plays an important role in defining the electrical resistivity of materials irradiated by intense, subpicosecond laser pulses, and that the resulting resistive magnetic field and feedback on the fast electron beam transport are strongly affected [13]. It was also shown that the fast electron transport pattern is largely defined by the target resistivity at low temperatures [14]. An annular fast electron beam transport pattern in Si was demonstrated to originate from a dip in the resistivity-temperature profile at a few $\mathrm{eV}[14,15]$.

In this Letter, we report on the first investigation of the effects of lattice melt on the properties of resistive magnetic fields and fast electron transport in solids, which are isochorically preheated using laser-accelerated protons. Lattice-order gradients are shown to strongly affect current propagation. Whereas the use of laser light to directly preheat a solid predominately heats the irradiated surface (due to absorption within the skin depth), and the use of $\mathrm{x}$ rays volumetrically heats it over an extended length, irradiation with a broad spectrum of laser-accelerated multi-MeV protons enables heating (and thus lattice-melt) gradients to be induced over the tens-of-microns depth at the fast electron source for which the resistive magnetic field is strongest. This enables the electrical resistivity, and thus properties of the magnetic field, to be varied for fixed fast electron source parameters. We thus demonstrate, for the first time, that the divergence, spatial-intensity profile, and symmetry of the fast electron beam can be actively manipulated by tailoring temperature and resistivity gradients within the target using radiation produced by a separate intense laser pulse. 
The experiment was performed using the Vulcan laser at the Rutherford Appleton Laboratory. Two beams of $p$ polarized, $1.053 \mu \mathrm{m}$-wavelength light were employed, in the arrangement shown in Fig. 1(a). The first, hereafter referred to as "beam 1," delivered pulses of duration $\tau_{1}=$ $10 \mathrm{ps}$ (full width at half maximum, FWHM), with maximum energy on target equal to $200 \mathrm{~J}$. These were focused to a diameter of $6 \mu \mathrm{m}$ (FWHM), onto a $20 \mu \mathrm{m}$-thick Au target, to produce a near-uniform beam of multi-MeV protons via rear-side sheath acceleration. The peak intensity was $I_{1}=2 \times 10^{19} \mathrm{~W} \mathrm{~cm}^{-2}$. Figure $1(\mathrm{~b})$ shows the energy spectrum and divergence of the proton beam, as characterized using a stack of dosimetry film (radiochromic film, RCF) $[13,16]$ [not shown in Fig. 1(a)]. The beam, with divergence shown in the inset, propagates over an axial distance of $1100 \mu \mathrm{m}$ to the main target sample, which was $200 \mu \mathrm{m}$-thick $\mathrm{Si}$, with $3 \mathrm{~mm} \times 3 \mathrm{~mm}$ lateral dimensions. The axis of the proton beam is at an angle of $45^{\circ}$ with respect to the Si target, to ensure any transmitted protons from this heating "pump" beam are directed away from the primary diagnostic stack of dosimetry film. The fast electron "probe" beam is produced by the second laser beam ("beam 2"), which delivered pulses with $\tau_{2}=1 \mathrm{ps}$ (FWHM) and a maximum energy (on-target) of $60 \mathrm{~J}$. The angle of incidence with respect to target normal was $15^{\circ}$ and the focal spot diameter was $5 \mu \mathrm{m}$ (FWHM), giving a calculated peak intensity $I_{2}=7 \times 10^{19} \mathrm{~W} \mathrm{~cm}^{-2}$. Changes to the transport properties of the fast electron beam within the target were diagnosed by measurement of the spatial-intensity distribution of the rear-side sheath-accelerated proton beam, using an RCF stack positioned $5 \mathrm{~cm}$ downstream. The diagnostic technique is described in fuller detail in Ref. [17].

The 1D hydrodynamic code HELIOS [18] was used to determine the heating profile within the $\mathrm{Si}$, as produced by the measured proton spectrum shown in Fig. 1(b). The calculations include the time-of-flight spreading of the
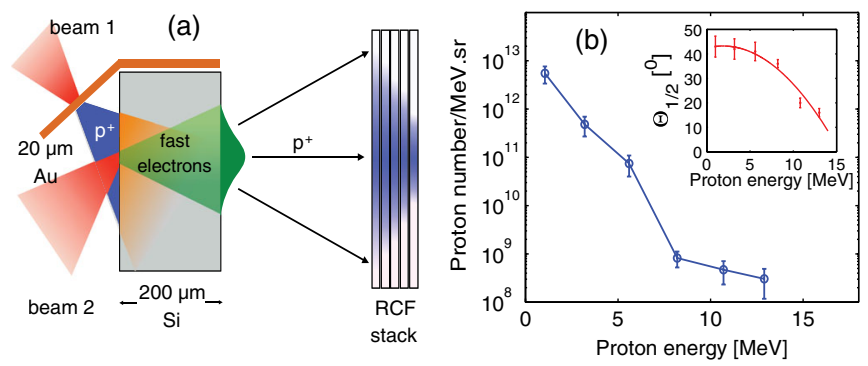

FIG. 1 (color online). (a) Schematic illustrating the experiment arrangement. Sheath-accelerated protons produced by the interaction of a pulse ( $200 \mathrm{~J}, 10 \mathrm{ps}$ ) from beam 1 with a $20 \mu \mathrm{m}$-thick $\mathrm{Au}$ foil are used to preheat and induce lattice melt in a $200 \mu \mathrm{m}$ thick Si target. Pulses (60 J, 1 ps) from beam 2 are used to drive fast electron generation and transport within the Si sample. (b) Experimentally measured proton spectrum produced by beam 1. The inset shows the proton beam divergence half-angle $\left(\Theta_{1 / 2}\right)$ as a function of energy. proton beam between the two targets and hence the arrival time as a function of proton energy. The resulting temperature-depth profile is shown in Fig. 2(a) at given times $t_{\text {heat }}$, after the arrival of the most energetic (i.e., $13 \mathrm{MeV}$ ) protons. A temporal separation of the two laser pulses equal to $30 \mathrm{ps}$ corresponds to $t_{\text {heat }}=10 \mathrm{ps}$. At this time only the low flux of high energy (10-13 MeV) protons contribute to the heating, resulting in a relatively low temperature. At $t_{\text {heat }}=30 \mathrm{ps}$, protons with energy above $2 \mathrm{MeV}$ have driven a sharp temperature rise to $\sim 2 \mathrm{eV}$ at depths of $0-50 \mu \mathrm{m}$, decreasing to $\sim 0.5 \mathrm{eV}$ at $100 \mu \mathrm{m}$. Heating for longer, with the high flux of lower energy protons, increases the temperature further, particularly within the first $20 \mu \mathrm{m}$ of the target. Heating with the full proton population results in an exponentially decreasing temperature profile, to around $0.5 \mathrm{eV}$ at $100 \mu \mathrm{m}$ depth.

When energy is deposited in a material, the electrons are quickly heated and over the course of picoseconds transfer energy, mediated by electron-phonon interactions, to the lattice ions, while the target remains at solid density $[19,20]$. The lattice-melt temperature for $\mathrm{Si}$ is $0.145 \mathrm{eV}$ [21] and the melting occurs over $\sim 5-18$ ps [22,23]. Thus, for $t_{\text {heat }} \sim 10 \mathrm{ps}$ and higher, lattice melt can be expected to depths of $\sim 100 \mu \mathrm{m}$.

Figure 2(b) shows the resistivity-temperature profiles for ordered (lattice) and disordered forms of $\mathrm{Si}$, as determined from quantum molecular dynamics simulations combined with broad-range Kubo-Greenwood calculations [24,25], using the approach outlined in Ref. [14]. The two resistivity profiles are significantly different at the sub- $10 \mathrm{eV}$ temperatures achievable by proton heating. Lattice melt gives rise to an order of magnitude increase in resistivity at a few $\mathrm{eV}$. The temperature profile induced by proton heating thus drives significant resistivity gradients in the target prior to the launch of the fast electron beam.
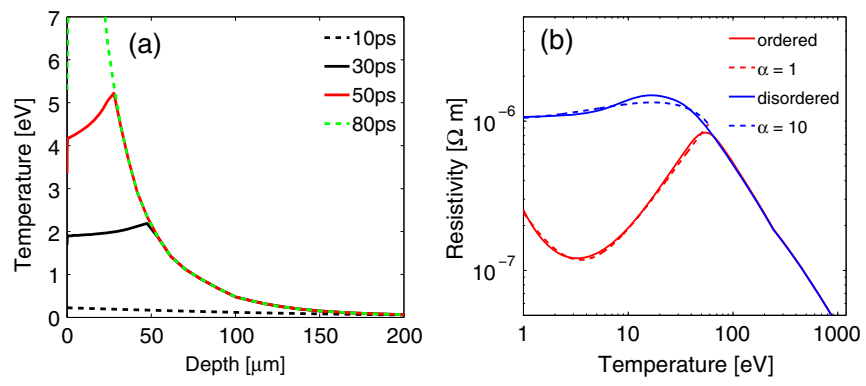

FIG. 2 (color online). (a) HELIOS hydrodynamic simulation results showing the temperature profile within the $\mathrm{Si}$ target resulting from the proton-driven heating, at given times $\left(t_{\text {heat }}\right)$ after the arrival of the most energetic protons. (b) Electrical resistivity as a function of temperature for ordered and disordered $\mathrm{Si}$, calculated via $a b$ initio quantum molecular dynamics simulations coupled with the Kubo-Greenwood equation (see main text). The dashed curves correspond to fits made to define a lattice order parameter $\alpha$. 
To investigate the influence that lattice-melt and thus resistivity gradients have on fast electron transport, the spatial-intensity distribution of the diagnostic proton beam (accelerated from the rear side of the Si target) is measured as a function of $t_{\text {heat }}$ and for reference unheated targets (beam 2-only shots). Representative results are shown in Fig. 3. Compared to the unheated reference, the most salient features of the heated target results are (i) the overall divergence of the proton beam is significantly increased, (ii) the beam profile changes from a center-peaked distribution to a strong annular beam, and, (iii) the asymmetry in the beam increases. These features are observed even for the shortest $t_{\text {heat }}=10 \mathrm{ps}$. This implies that significant energy coupling to the lattice ions has occurred on this time scale. Note that the unheated reference result is in excellent agreement with results from a different experimental investigation into fast electron transport in (unheated) $\mathrm{Si}$ at a similar peak intensity of $\sim 7 \times 10^{19} \mathrm{~W} \mathrm{~cm}^{-2}$ [14].

To explore the underlying physics, simulations of fast electron transport were performed using the particle-based 3D-hybrid code ZEPHYROS [9]. The code was revised to enable a transition between two resistivity-temperature profiles, corresponding to the ordered and disordered profiles in Fig. 2(b), to be implemented across the simulation grid. This was achieved by introducing a parameter $\alpha$ to assign a specific degree of lattice structure to each cell, where the limits $\alpha=1$ and $\alpha=10$ correspond to the ordered and disordered resistivity-temperature curves, respectively. The model curves for both limits are included in Fig. 2(b). In high temperature regions (for example, to a depth of $50 \mu \mathrm{m}$ for $t_{\text {heat }}=30 \mathrm{ps)} \alpha$ is set to 10 and in regions for which proton heating does not raise the temperature above the $\mathrm{Si}$ lattice-melt temperature of $0.145 \mathrm{eV}$ it is set to 1 . The value between these limits varies in an exponential profile matched to the HELIOS preheat profile [Fig. 2(a)]. Modeling the detailed lattice-melt dynamics induced within the target is beyond the scope of this investigation. This
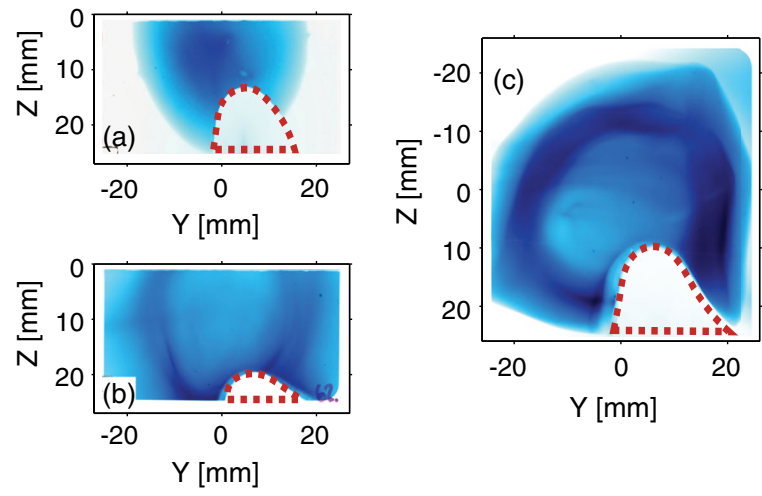

FIG. 3 (color online). Example measured proton spatial-dose distributions (at $3.3 \mathrm{MeV}$ ) for peak laser intensity equal to $7 \times 10^{19} \mathrm{~W} \mathrm{~cm}^{-2}$, for (a) unheated, and after (b) $10 \mathrm{ps}$, and (c) $30 \mathrm{ps}$ of proton heating. The region bounded by the dashed red curve was blocked. simplified prescription based on the proton-induced heating profile is adequate to explore the effects of gradients in the lattice order on fast electron transport.

The simulations were performed on a $200 \mu \mathrm{m} \times 400 \mu \mathrm{m} \times$ $400 \mu \mathrm{m}$ grid, with a cell resolution of $\Delta X=\Delta Y=$ $\Delta Z=1 \mu \mathrm{m}$. The fast electron population was injected at $[X, Y, Z]=[0,0,0]$. The laser-to-fast electron energy conversion factor was set to 0.3 , with a laser pulse duration of $1 \mathrm{ps}$, wavelength equal to $1 \mu \mathrm{m}$ and focal spot diameter $d_{L}=5 \mu \mathrm{m}$, resulting in peak laser intensity $I_{L}=7 \times 10^{19} \mathrm{~W} \mathrm{~cm}^{-2}$. The electrons propagate in the $X$ direction with an exponential energy distribution, with mean temperature $T_{f}$ given by ponderomotive scaling [26], and are injected with a uniform angular distribution over a cone subtended by a half-angle of $50^{\circ}$ [27]. With the exception of the inclusion of the lattice order- and thus resistivity-gradients all other initial parameters are the same for the simulations discussed below.

Figure 4 shows the $2 \mathrm{D}[X-Y]$ midplane resistivity and magnetic field profiles (after $1.4 \mathrm{ps}$ ) for three example cases: (a) initially cold, ordered $\mathrm{Si}$; (b) an initial resistivity gradient in the $X$ direction only (i.e., assuming a heating beam moving into the target in the $+X$ direction); and (c) resistivity gradients in both the $X$ and $Y$ directions, as driven by a heating beam irradiating the target at a $45^{\circ}$ angle, as in the experiment [see Fig. 1(a)]. As results are shown $1.4 \mathrm{ps}$ after the start of the fast electron beam, the effects of self-induced heating (and thus additional selfinduced resistivity changes) due to the collisional return current drawn by the fast electron beam are also observed.

One of the most pertinent features of the results is that although in all three cases an azimuthal magnetic field
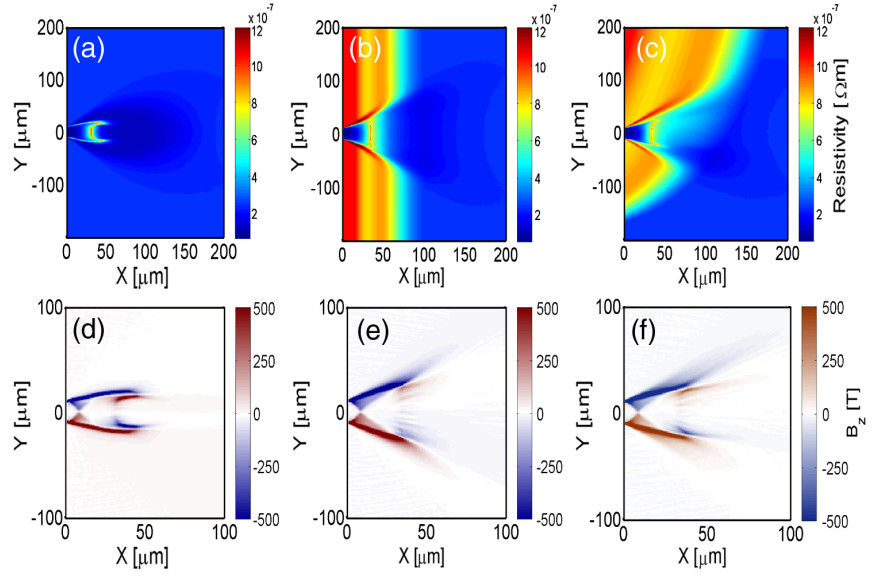

FIG. 4 (color online). Hybrid-PIC simulation results, 1.4 ps after the start of the beam 2 laser pulse, showing the $[X-Y]$ midplane 2D map of resistivity $\eta$ (in units of $\Omega \mathrm{m}$ ) for (a) initially unheated (i.e., ordered), (b) lattice-melt gradient from the $X=0$ boundary, and (c) lattice-melt gradient at $45^{\circ}$. The corresponding $[X-Y]$ midplane 2D maps of magnetic flux density ( $B_{Z}$ component in Tesla) are shown in (d), (e), and (f), respectively, enlarged to show the detail of the magnetic field. 
grows, which results in a pinching of the beam towards the axis, the radius of the field at a depth of $20-50 \mu \mathrm{m}$ is significantly different between the ordered and meltgradient cases. The pinching effect is stronger in the former, giving rise to a less divergent electron beam. The sharp resistivity gradients in this case are induced by the heating of the target driven by the fast electron beam and the resulting changes in resistivity given by the ordered resistivity-temperature curve in Fig. 2(b). The high resistivity at the edges of the beam and on-axis at $X=40 \mu \mathrm{m}$ correspond to temperatures of $\sim 50 \mathrm{eV}$, at which the ordered resistivity-temperature profile peaks. In cases (b) and (c) the resistivity is already high for $X<50 \mu \mathrm{m}$ due to the disorder induced by lattice melt. A slight increase is still driven at the beam edge due to the broad peak at $\sim 20 \mathrm{eV}$ [Fig. 2(b)], and the global effect is that the resistivity onaxis (due to the Spitzer regime) is considerably lower than the region surrounding the beam. Because of the $\nabla \eta \times \mathbf{j}_{f}$ dependency of the $B$-field growth, the localized pinching effect of the field is significantly reduced, leading to an overall increase in beam divergence. A second feature to note is that the strength of the reversed magnetic field just inside the beam envelope, as clearly observed in Fig. 4(d), and identified in Ref. [14] as arising from the dip at $\sim 3 \mathrm{eV}$ in the ordered Si resistivity-temperature profile in Fig. 2(b), is significantly weaker. This is because the corresponding dip for the case of disordered $\mathrm{Si}$ is minimal. When comparing Figs. 4(e) and 4(f), we find that when the heating is induced at an angle, the asymmetry in the resistivity distribution about the $Y=0$ axis gives rise to a small asymmetry in the $B$-field.

The corresponding fast electron transport results for the cases with ordered Si [Fig. 4(a)] and for the lattice melt gradient at $45^{\circ}$ [Fig. 4(c)] are shown in Fig. 5. In agreement with results reported in Ref. [14] (for a similar laser intensity), the electron beam distribution in the initially cold, ordered target is peaked on-axis with a Gaussian-like profile. The reversal in the magnetic field just inside the beam envelope shown in Fig. 4(d) has little effect at this peak laser intensity due to the overall strong pinching of the main enveloping $B$-field. The beam pattern obtained in the case with the initial resistivity gradient is, however, significantly different. The beam diameter (FWHM) is almost a factor of 2 larger and contains a clear annular structure, as shown in Fig. 5(e). Moreover, the asymmetry in the resistivity and resultant $B$-field gives rise to an asymmetry in the annular electron transport pattern, as evidenced by the horizontal line-out displayed in Fig. 5(e). For the case of axial symmetry in the resistivity gradient (not shown), we find that the electron annular distribution is symmetrical. Figures 5(c) and 5(f) correspond to the calculated proton beam profiles, as obtained by mapping the fast electron density maps at the target rear surface into $E$-field distributions and, subsequently, into an expanding proton front. The principles behind this approach are

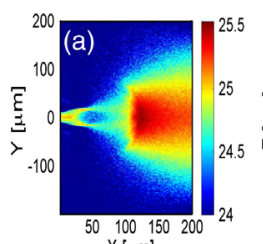

$$
X[\mu \mathrm{m}]
$$
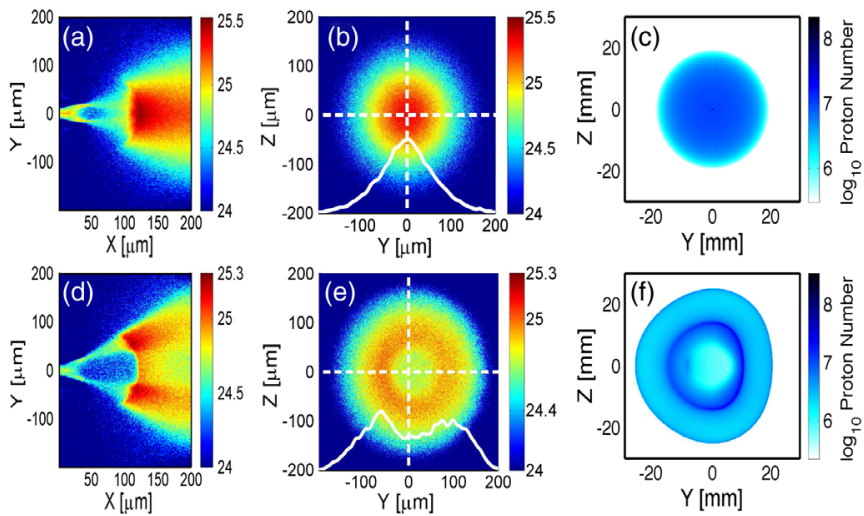

FIG. 5 (color online). 3D hybrid-PIC simulation results showing $\log _{10}$ fast electron density maps $\left(\mathrm{m}^{-3}\right)$, in the $[\mathrm{X}-\mathrm{Y}]$ midplane and rear surface [Y-Z] plane, 1.4 ps and 1.6 ps after laser irradiation respectively, for the case of: (a) and (b) initially unheated Si (i.e., ordered lattice); and (d) and (e) initial heating and disorder gradients at $45^{\circ}$; all for peak intensity equal to $7 \times 10^{19} \mathrm{~W} \mathrm{~cm}^{-2}$. (c) and (f) are the corresponding modeled proton-spatial intensity maps arising from the rear-surface density profiles in (b) and (e), respectively.

outlined in [17], and the present modeling takes account of the evolution of the electron density distribution at the target rear. The final proton beam distributions resulting from the fast electron transport simulation results are in good qualitative agreement with the experiment results in Fig. 3. Even the asymmetry in the proton beam, resulting from heating the target at an angle, is reproduced.

Our hybrid-PIC modeling demonstrates, for the first time, that gradients in electrical resistivity produced via spatial variation in lattice melt strongly affect the properties of fast electron transport in solids. Experimentally, the use of laser-driven protons to isochorically induce temperature gradients on tens-of-picoseconds time scales enables lattice melt and, thereby, resistivity gradients and resistive magnetic fields to be actively controlled. This in turn enables dynamic manipulation of the transport of mega-ampere currents of energetic electrons in homogenous targets.

Previous approaches to inducing resistive guiding of fast electrons have involved either prefabricating the target with high and low resistivity materials (in a core-cladding arrangement [6] or in narrow filaments with radialdependent density [11]), or by the choice of metal target material and laser-drive parameters to induce desired spatial variations in ionization [12]. Our approach demonstrates resistive guiding without the need for complex target manufacture and with less constraints on target choice. Using variations of the approach introduced here, the beam divergence, spatial-intensity pattern, and symmetry could potentially all be altered on a shot-to-shot basis, without complex target engineering. The use of masks and filters in the proton heating beam could be used to produce more complex heating and resistivity patterns. This may enable the exploration of new concepts such as magnetic mirrors 
for electron transport [28], but with tunable focusing and guiding properties.

We acknowledge the expertise of the Central Laser Facility staff and computing resources provided by STFC's e-Science project. This work is financially supported by EPSRC (Grants No. EP/J003832/1, No. EP/ L001357/1, and No. EP/K022415/1). The research leading to these results has also received funding from LASERLAB-EUROPE (Grant No. 284464, EC's Seventh Framework Programme) and from the Air Force Office of Scientific Research, USAF (Grant No. FA865513-1-3008).

*paul.mckenna@strath.ac.uk

[1] M. Tabak, J. Hammer, M. E. Glinsky, W. L. Kruer, S. C. Wilks, J. Woodworth, E. M. Campbell, M. D. Perry, and R. J. Mason, Phys. Plasmas 1, 1626 (1994).

[2] M. D. Perry, J. A. Sefcik, T. Cowan, S. Hatchett, A. Hunt, M. Moran, D. Pennington, R. Snavely, and S. C. Wilks, Rev. Sci. Instrum. 70, 265 (1999).

[3] X. H. Yuan et al., New J. Phys. 12, 063018 (2010).

[4] H. Chen et al., Phys. Rev. Lett. 105, 015003 (2010).

[5] A. R. Bell, A. P. L. Robinson, M. Sherlock, R. J. Kingham, and W. Rozmus, Plasma Phys. Controlled Fusion 48, R37 (2006).

[6] A. P. L. Robinson and M. Sherlock, Phys. Plasmas 14, 083105 (2007).

[7] S. Kar, A. Robinson, D. Carroll, O Lundh, K. Markey, P. McKenna, P. Norreys, and M. Zepf, Phys. Rev. Lett. 102, 055001 (2009).

[8] B. Ramakrishna et al., Phys. Rev. Lett. 105, 135001 (2010).
[9] A. P. L. Robinson, M. H. Key, and M. Tabak, Phys. Rev. Lett. 108, 125004 (2012).

[10] H. Schmitz, R. Lloyd, and R. G. Evans, Plasma Phys. Controlled Fusion 54, 085016 (2012).

[11] A. Debayle, L. Gremillet, J. J. Honrubia, and E. d'Humières, Phys. Plasmas 20, 013109 (2013).

[12] Y. Sentoku, E. d'Humières, L. Romagnani, P. Audebert, and J. Fuchs, Phys. Rev. Lett. 107, 135005 (2011).

[13] P. McKenna et al., Phys. Rev. Lett. 106, 185004 (2011).

[14] D. A. MacLellan et al., Phys. Rev. Lett. 111, 095001 (2013).

[15] D. A. MacLellan, D. C. Carroll, R. J. Gray, A. P. L. Robinson, M. P. Desjarlais, D. Neely, and P. McKenna, Plasma Phys. Controlled Fusion 56, 084002 (2014).

[16] J. Fuchs et al., Phys. Rev. Lett. 91, 255002 (2003).

[17] D. A. MacLellan, D. C. Carroll, R. J. Gray, N. Booth, B. Gonzalez-Izquierdo, H. W. Powell, G. G. Scott, D. Neely, and P. McKenna, Laser Part. Beams 31, 475 (2013).

[18] J. J. MacFarlane, I. E. Golovkin, and P. R. Woodruff, J. Quant. Spectrosc. Radiat. Transfer 99, 381 (2006).

[19] A. Mancic et al., Phys. Rev. Lett. 104, 035002 (2010).

[20] A. Pelka et al., Phys. Rev. Lett. 105, 265701 (2010).

[21] C. J. Glassbrenner and G. A. Slack, Phys. Rev. 134, A1058 (1964).

[22] M. D. Kluge, J. R. Ray, and A. Rahman, J. Chem. Phys. 87, 2336 (1987).

[23] C. V. Shank, R. Yen, and C. Hirlimann, Phys. Rev. Lett. 50, 454 (1983).

[24] G. Kresse and J. Hafner, Phys. Rev. B 47, 558 (1993).

[25] M. P. Desjarlais, J. D. Kress, and L. A. Collins, Phys. Rev. E 66, 025401 (2002).

[26] S. C. Wilks and W. L. Kruer, IEEE J. Quantum Electron. 33, 1954 (1997).

[27] M. Coury et al., Phys. Plasmas 20, 043104 (2013).

[28] A. P. L. Robinson and H. Schmitz, Phys. Plasmas 20, 062704 (2013). 